\title{
Fundamentos y clasificación de las eflorescencias en ladrillos de construcción
}

\author{
Basis and classification of efflorescences in \\ construction bricks
}

Fecha de recepción: $21-\mathrm{I}-00$

Fecha de aceptación: 9-VI-00
J.Ma.RINCÓN YM.ROMERO

Instituto de Ciencias de la Construcción Eduardo Torroja-CSIC

ESPAÑA

\section{RESUMEN}

El problema de las eflorescencias en productos de tierra cocida y en concreto en los ladrillos de construcción, constituye una patología que disminuye la calidad estética de los edificios. Sus tipos y causas son muy diversas y sólo una revisión, de todas las clases y variables que influyen en la aparición de eflorescencias, puede permitir empezar a comprender este fenómeno. Fundamentalmente es la migración de sales solubles de distinta procedencia la responsable de esta patología. Se muestran, de una manera directa y resumida, los principios básicos de formación de eflorescencias, con ejemplos de las más frecuentes.

\section{SUMMARY}

The efflorescence problem in clay products and more specifically in construction bricks is a pathology which produce a decrease in the aesthetic quality of buildings. Their types and causes are very different and only a review of all the variables and classification can allow to start the understanding of this phenomenon. Basically, the migration of soluble salts from different sources is the responsible of this pathology. Here is shown directly and briefly the main principles of efflorescece formation with some of the most frequent examples.

\section{INTRODUCCIÓN}

Las eflorescencias en ladrillos consisten en la aparición de manchas blancas debidas a depósitos salinos, que suelen aparecer en su superficie, cualquiera que sea su origen, en un momento determinado.

El agua o humedad que circula por la red capilar del ladrillo contiene disueltas sales, fundamentalmente sulfatos, que pueden alcanzar la superficie del ladrillo y precipitarse en ella como manchas blancas más o menos extendidas.
Este defecto no sólo afecta a la estética y aspecto de la superficie del edificio construido con el ladrillo eflorescente, sino que también puede afectar gravemente, en algunos casos, a la "vida útil" del ladrillo, que puede llegar a desmoronarse con el tiempo.

Se puede decir claramente en palabras de A. GarcíaVerduch, como investigador pionero del estudio de las eflorescencias en nuestro país, que "un ladrillo eflorescente es un ladrillo enfermo" (1).

En 1925, Jackson (2) definió la eflorescencia como "la formación de un depósito de sales minerales solubles, 
sobre la superficie de una pieza cerámica terminada, por exposición a los agentes atmosféricos".

Dado que las sales se depositan en la superficie por estar ésta más expuesta a la evaporacion, resulta que los ladrillos con mayor porosidad o texturas más abiertas son los que tienen mayor tendencia a eflorescer. De manera que cuando un ladrillo presenta una capacidad de absorción de agua nula, prácticamente no presenta eflorescencias.

En algunos casos se usa la palabra "eflorescencia" en sentido general designando todos los casos de cristalización de sales solubles y se usa más en concreto el término "eflorescencia" para el depósito cristalino que se forma en la superficie. Cuando la cristalización salina tiene lugar en el interior del material es más correcto usar la palabra "criptoeflorescencia". Antiguamente, las eflorescencias que se formaban por la descomposición en el suelo de material orgánico conteniendo nitrógeno o por defectuosos sistemas de alcantarillado en las edificaciones antiguas se designaban con los nombres de "salitre" o "nitro de pared". En la actualidad es raro que estas sales nitrogenadas aparezcan en obras de ladrillos y, en tal caso, sólo pueden aparecer en cercanías de establos o estercoleros.

En general, aunque más adelante se entrará en más detalles, por orden de frecuencia, las eflorescencias están constituidas por sulfatos de sodio, calcio, potasio y magnesio, cloruros y nitratos alcalinos. Estas sales aparecen en la superficie del ladrillo tanto después del secado como de la cocción de las piezas. Después de la cocción pueden aparecer también sulfo-aluminatos $\mathrm{y}$ carbonatos alcalinos (3).

\section{PROCESOS FÍSICOS QUE AFECTAN A LA APARICIÓN DE EFLORESCENCIAS}

En el complejo proceso de aparición de eflorescencias influyen distintos fenómenos físico-químicos, tales como (4):

(1) Equilibrios químicos de las disoluciones de sales.

(2) Distribución heterogénea de las sales solubles en

el interior del material.

(3) Estructura de la red capilar de porosidad.

(4) Grado de saturación acuosa de la red de poros.

(5)Configuración geométrica del conjunto y microestructura de la porosidad .

(6) Condiciones ambientales de humedad, temperatura, velocidad del aire, etc...

A pesar de lo espectacular del fenómeno eflorescente, el peso de las sales de una eflorescencia sobre el total de la masa del ladrillo representa sólo una pequeña cantidad, menor de $10^{-6}-10^{-8} \mathrm{~g}$, del peso de la pared del edificio. Sin embargo, las cuestiones que plantea este fenómeno tienen a veces difícil respuesta y dan lugar a casos contradictorios como: ¿por qué las eflorescencias aparecen más en ladrillos de muros o paredes que en ladrillos ? o ¿por qué ladrillos que eflorescen en obra, sin embargo no tienen tendencia a hacerlo en el laboratorio? ... por citar sólo dos ejemplos.

Analicemos con más detalle algunos de los procesos físicos que pueden influir en la formación de eflorescencias:

1) La capacidad de absorción de agua (CAA) del ladrillo es uno de los factores más determinantes a la hora de la formación de eflorescencias, ya que condiciona, de hecho, la concentración de sales solubles que pueden alcanzarse dentro de la red capilar interior del ladrillo. La CAA influye además sobre el tiempo que tarda en aparecer la eflorescencia. Ya se ha indicado anteriormente que los ladrillos con elevada CAA eflorescen más rápido que los de baja CAA. Parece ser que el valor mínimo para producirse eflorescencias es de una CAA $=6 \%$ (1). Asimismo, las bajas temperaturas, sol y rachas de viento pueden favorecer la aparición de este defecto en paredes o muros, incidiendo este fenómeno especialmente en primavera.

2) La microestructura (tamaño y forma) de la porosidad, así como la textura de las fases cristalinas que precipitan en los capilares tiene también gran importancia. Por ejemplo, el sulfato sódico efloresce más fácilmente que el sulfato potásico, y el sulfato magnésico precipita con mucha facilidad.

3) La distribución de sales solubles en el ladrillo recién salido del horno condiciona también la posterior formación de eflorescencias. Generalmente se analizan las sales sobre el ladrillo molido por lo que no se tiene más información de la distribución de las mismas. Por tanto, ya que hoy en día es posible disponer de la técnica de microscopía electrónica de barrido, MEB/EDX, la distribución de sales debería analizarse no sólo entre la superficie y el interior, sino también a lo largo de todo el área transversal del ladrillo. De hecho, cuando la eflorescencia se forma por reacción entre los gases sulfurados del horno y las arcillas con que se moldeó el ladrillo, estas diferencias son importantes en ladrillos calizos de cocción blanca o amarillenta.

La manera de realizar el mojado y secado del ladrillo en obra afecta también a la formación de eflorescencias. Así, la eflorescencia es menos probable cuando el agua entra en el ladrillo por la misma cara por la que después ha de salir por evaporación. Si el agua entra por caras distintas a la de evaporación, la cristalización comienza a poco de iniciarse el secado debido a la progresiva sobresaturación 
en sales solubles de la red capilar. Por esta razón, las eflorescencias aparecen siempre con mucha frecuencia en obras nuevas debido a que los ladrillos suelen mojarse por todas sus caras antes de ser colocados en obra. Así, pues, los ladrillos no deberían mojarse antes de su colocación. Solamente deberían mojarse cuando la CAA es muy grande o para facilitar un mejor agarre por el mortero de cemento, cuando tienen la superficie sucia. En cualquier caso, el mojado debe ser muy breve.

\section{COMPOSICIÓN DE SALES SOLUBLES}

La composición de las sales que dan lugar a las eflorescencias se conoce desde años. El sulfato sódico, $\mathrm{SO}_{4} \mathrm{Na}_{2}$, es la sal más frecuente y ya en 1877 (1) se sabía de ladrillos que contenían un $42 \%$ de dicha sal. Desde entonces se han encontrado toda clase de sulfatos, cloruros y nitratos en ladrillos, así como compuestos de $\mathrm{V}$, Mo, Cr, Ni y Mn.

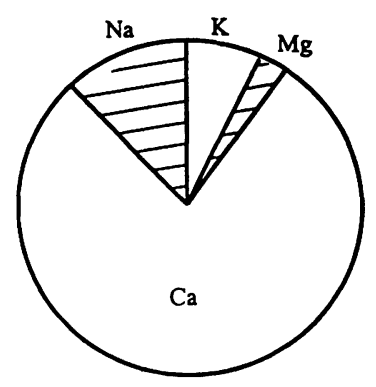

Sales extraidas del ladrillo

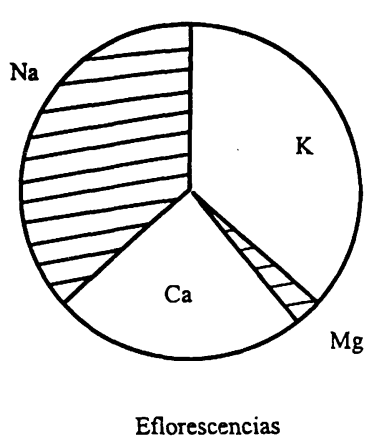

Eflorescencias
Figura 1.- Descomposición de la pirita y formación de sulfatos (según (5)).
Es necesario destacar que las sales que aparecen en la superficie del ladrillo suelen tener composición diferente de las del interior. La Figura 1 muestra un diagrama de la proporción relativa de cationes que suelen aparecer en las eflorescencias de sulfato (1).

Se confirma, pues, por varios autores (1) (5) que el $\mathrm{SO}_{4} \mathrm{Ca}$ suele aparecer fundamentalmente en el interior del ladrillo. Este hecho tiene que ver con la diferente solubilidad de las sales ya que $\mathrm{SO}_{4} \mathrm{Na}_{2}, \mathrm{SO}_{4} \mathrm{~K}_{2}$ y $\mathrm{SO}_{4} \mathrm{Mg}$ son mucho más solubles que el $\mathrm{SO}_{4} \mathrm{Ca}$, por lo que se movilizan más fácilmente hacia la superficie.

El principal factor que interviene en la formación de eflorescencias tiene que ver con la composición básica de las materias primas con las que se prepara la pasta original, así como las posibles sustancias dañinas que pueden contener. Es conocido que la presencia de cal, sales solubles o pirita en la materia prima condiciona la composición de las sales eflorescentes. La Figura 2 muestra un esquema de estos componentes dañinos y su efecto sobre la composición de las eflorescencias e incluso el daño que pueden provocar a las propiedades del material (5).

Los principales componentes que pueden producir daños en los ladrillos son cal libre, sales solubles y pirita. Tanto si estas sustancias están presentes en las materias primas como en la pasta o mezcla inicial, pueden dar lugar a deformaciones y defectos en el producto final. Más adelante, en el apartado de Normas y Ensayos, se expondrán los tipos de análisis que pueden realizarse para controlar estos componentes en las materias primas.

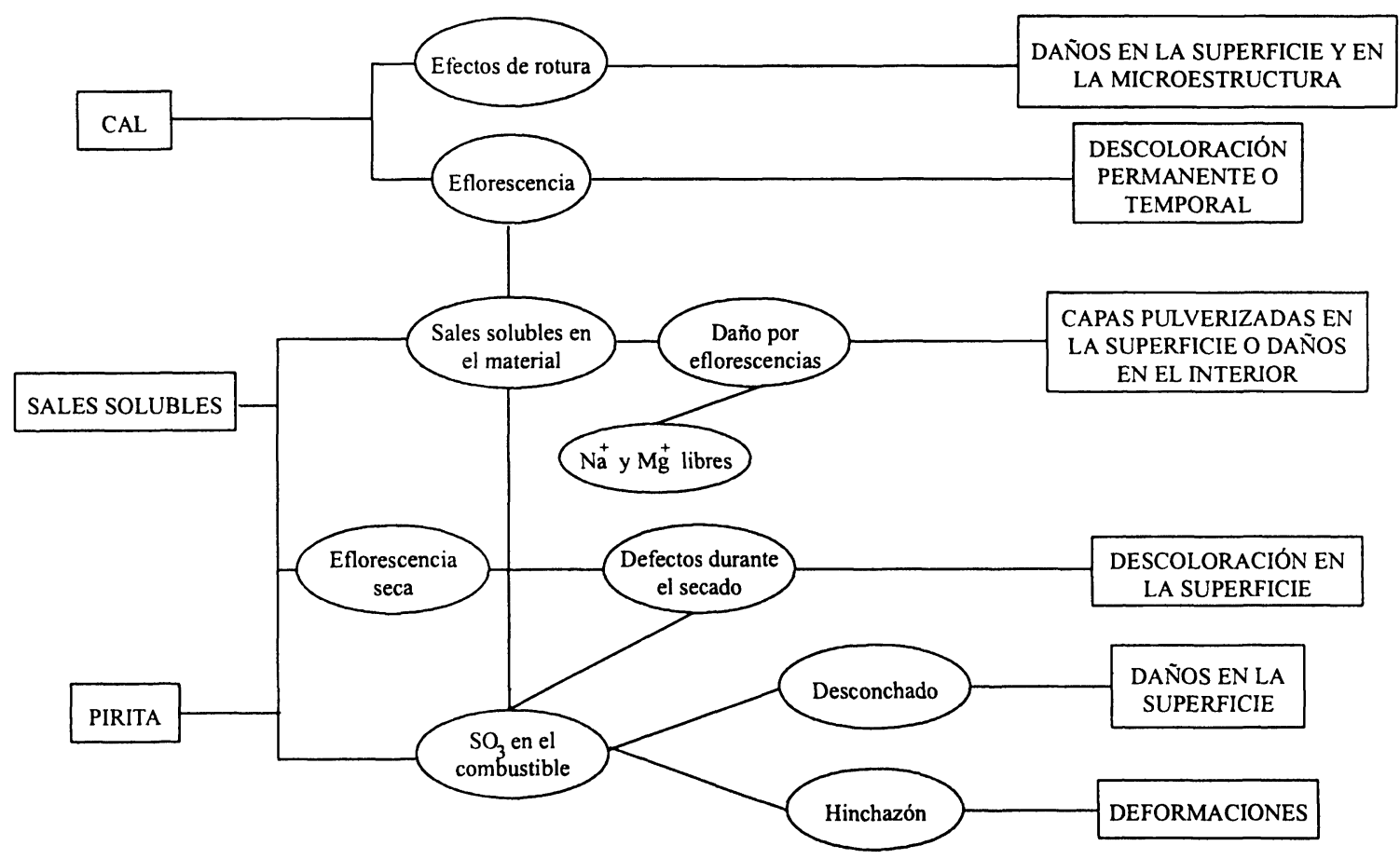

Figura 2.- Componentes de las materias primas (modificado de la referencia 5). 


\section{TIPOS DE EFLORESCENCIAS}

Las eflorescencias pueden formarse en un ladrillo en diversas etapas de la vida del mismo, a saber: después de fabricado, una vez instalado en obra o bien aparecer hasta en las materias primas originales. Por tanto, desde este punto de vista del momento o lugar de su aparición, se pueden distinguir los siguientes tipos de eflorescencias:

* Velo de secado

* Velo de secadero

* Velo de horno

* Velo de obra

VELO DE SECADO Se llama así a la eflorescencia o mancha blanca que aparece cuando se seca al aire una masa húmeda de arcilla cruda que contiene sales solubles.

VELO DE SECADERO Es la eflorescencia que aparece en la masa cruda o verde, cuando la arcilla, una vez amasada y moldeada, se somete al proceso de secado previo a la cocción. En este caso no se trata de un fenómeno natural como el anterior que depende únicamente de la masa arcillosa. Ahora la aparición de eflorescencia puede estar además influida por la atmósfera del secadero.

VELO DE HORNO Es la eflorescencia que aparece una vez cocido el ladrillo por aparición de la denominada "capa de reacción" que se produce por la interacción entre la capa salina original y los componentes composicionales y microestructurales del ladrillo. Así pues, en su formación influye aparte de la composición química, la textura del mismo. Las interaciones de la atmosfera del horno, especialmente si contiene vapores de $\mathrm{SO}_{2}$, son también responsables de la aparición de este efecto a la salida del horno.

VELO DE OBRA Hemos denominado con este nombre por vez primera a la eflorescencia más comúnmente observada, que es la que aparece en las paredes de los edificios una vez construidos. En estas eflorescencias, las sales se hacen visibles al ser transportadas por el agua o la humedad desde el interior hacia el exterior del ladrillo, precipitándose en su superficie.

A continuación se comentan algunos detalles adicionales que influyen en la aparición de estos efectos. Así, se ha observado (4) que el tipo de proceso de moldeado puede afectar a la aparición del velo de secado, de manera que las piezas que se moldean en proceso semiseco no presentan este tipo de eflorescencias. La explicación se debe a que el agua contenida en el interior de la pieza está, en este caso, aislada por zonas, de manera que no puede ejercer su labor de transporte de sales hacia la superficie. La velocidad de secado puede afectar también a la aparición de este tipo de defecto. Así, cuando los ladrillos se secan a elevadas velocidades de secado y a mayor temperatura, el velo de secado se aprecia menos y/o está distribuido de forma más homogénea. Por el contrario, si la velocidad de secado es lenta, la eflorescencia se forma más en los vértices y aristas del ladrillo.

Respecto al velo de horno, consecuencia de la consolidación del velo de secado después de cocer la pieza, suele ser de color blanco, rosa o amarillento y generalmente de composición muy compleja. Si se produce por sales cálcicas puede ser de anortita, $\mathrm{CaO} \cdot \mathrm{Al}_{2} \mathrm{O}_{3} \cdot 2 \mathrm{SiO}_{2}$ (anortita). $\mathrm{Si}$ interviene el sodio, puede ser de labradorita, fase formada por solución sólida de albita $\left(\mathrm{Na}_{2} \mathrm{O} \cdot \mathrm{Al}_{2} \mathrm{O}_{3} \cdot 6 \mathrm{SiO}_{2}\right)$ en anortita. Se pueden llegar a formar incluso otros silicatos como cordierita, $2 \mathrm{MgO} \cdot 2 \mathrm{Al}_{2} \mathrm{O}_{3} \cdot 5 \mathrm{SiO}_{2}$, si el velo está producido por sales magnésicas.

\section{ORIGEN DE LAS EFLORESCENCIAS}

Las sales solubles que son inherentes al ladrillo pueden proceder de:

a) Presencia en las materias primas originales.

b) Formación durante el secado y cocción por reacción con gases circundantes.

c) Sales de residuos industriales, escombreras, etc...

d) Nitratos de la descomposición en el suelo de materias orgánicas nitrogenadas.

e) Sales de aguas marinas.

f) Sales que se forman al lavar los ladrillos con ácidos.

g) Sales procedentes de productos de limpieza.

La lista anterior es una enumeración en términos generales. A continuación se analizan con más detalle los orígenes más frecuentes de las eflorescencias, a saber:
A) Suelo
B) Ladrillo
C) Mortero

A. Suelo. Es frecuente encontrar suelos con altos contenidos en sulfatos de $\mathrm{Ca}, \mathrm{Na}$ y $\mathrm{Mg}$. En general los terrenos esquistosos arcillosos suelen estar muy contaminados por sales. Esta contaminación también se da en terrenos urbanos que contienen escorias, escombros o residuos industriales.

Cuando la eflorescencia aparece en el exterior del muro no suele tener más consecuencias que las simplemente estéticas, pero cuando hay "criptoeflorescencias" las sales se acumulan en el interior, pudiendo dar lugar a consecuencias catastróficas.

B. Ladrillo. Las sales solubles en el ladrillo pueden proceder de las materias primas originales o formarse, 
por reacción con los gases del horno, durante las etapas de secado y cocción. Otra posibilidad es que se formen por interacción de los distintos componentes de las materias primas. Estas sales solubles derivan del azufre que esté contenido en las materias primas, como:

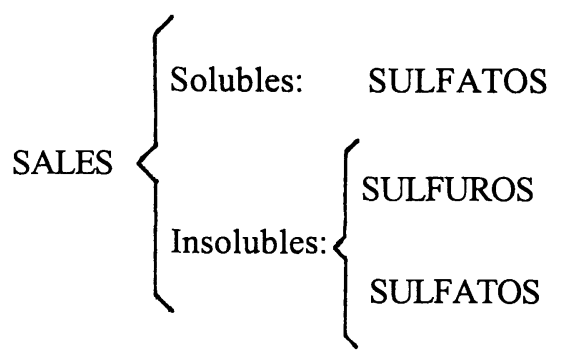

Las sales insolubles son las que normalmente dan lugar a eflorescencias. Ahora bien, la composición de las eflorescencias que aparecen en el ladrillo no tiene por qué ser de la misma composición que las existentes en las materias primas originales. Así, por ejemplo, puede darse el caso de un esquisto que no presente eflorescencias y sólo contenga $\mathrm{CO}_{3} \mathrm{Ca}$, cuando se le añada en crudo $\mathrm{SO}_{4} \mathrm{Mg} \cdot 7 \mathrm{H}_{2} \mathrm{O}$ de lugar a eflorescencias de $\mathrm{SO}_{4} \mathrm{Na}_{2}$ y $\mathrm{SO}_{4} \mathrm{Ca}$ en los ladrillos. Si se añadiera yeso, se producirían, en cambio, eflorescencias de $\mathrm{SO}_{4} \mathrm{Ca}, \mathrm{SO}_{4} \mathrm{Na}_{2}$ y algo de $\mathrm{SO}_{4} \mathrm{~K}_{2}$. De igual forma, si se añadiera pirita (sulfuro de hierro) se producirían abundantes eflorescencias de $\mathrm{SO}_{4} \mathrm{Mg},\left(\mathrm{SO}_{4}\right)_{2} \mathrm{AlK}, \mathrm{SO}_{4} \mathrm{Na}_{2}$ y $\mathrm{SO}_{4} \mathrm{Ca}$. Así pues, puede comprenderse con este ejemplo lo complicado que a veces puede resultar conocer el origen del fenómeno de formación de eflorescencias.

La presencia de gases, tales como $\mathrm{SO}_{2}$, en los secadores $\mathrm{y}$ hornos tiene mucho que ver con la formación de eflorescencias. Si la atmósfera sulfurosa actúa sobre la arcilla cuando ésta está aún húmeda puede favorecer la formación de eflorescencias. Además, si las arcillas contienen $\mathrm{CO}_{3} \mathrm{Ca} / \mathrm{o} \mathrm{CO} \mathrm{Cg}_{3} \mathrm{Mg}$ es más fácil que aumente su contenido en sulfatos en atmósferas sulfurosas. La Figura 3 presenta la absorción de $\mathrm{SO}_{3}$ de los gases de cocción comparativamente en una arcilla libre de cal con otra que contiene este mineral (5). Puede comprobarse que la máxima absorción de $\mathrm{SO}_{3}$ tiene lugar aproximadamente a $500^{\circ} \mathrm{C}$.

En general, la descomposición de las sales insolubles a altas temperaturas afecta a la formación de eflorescencias. Así, la pirita, $\mathrm{S}_{2} \mathrm{Fe}$, se descompone casi completamente a $600^{\circ} \mathrm{C}$ (Figura 4) (5), favoreciendo la cristalización de $\mathrm{SO}_{4} \mathrm{Mg}$ a partir del $\mathrm{SO}_{3}$ formado. Este sulfato tiene un intervalo de formación entre $300 \mathrm{y}$ $900^{\circ} \mathrm{C}$. Se puede observar que al disminuir el contenido de dicho sulfato se produce un aumento simultáneo en la formación de $\mathrm{SO}_{4} \mathrm{Ca}$ hasta altas temperaturas. $\mathrm{El} \mathrm{SO}_{4} \mathrm{Ca}$ no empieza a descomponerse hasta $1.000^{\circ} \mathrm{C}$. Aun así, estos procesos son muy relativos pues dependen de multitud de factores, tales como tamaño de partícula, resistencia del producto, tipo de pieza, atmósfera de cocción, velocidad de flujo de los gases, etc.

En otros ensayos se ha comprobado que mientras el $\mathrm{SO}_{4} \mathrm{Ca}$ se descompone en atmósfera oxidante entre 1300 y $1.400^{\circ} \mathrm{C}$, esta descomposición puede tener lugar entre 700 y $800^{\circ} \mathrm{C}$ en atmósfera reductora. Hasta es posible que en atmósfera de $\mathrm{SO}_{2}$ no se descompongan el $\mathrm{SO}_{4} \mathrm{Mg}$ y el $\mathrm{SO}_{4} \mathrm{Na}_{2}$ a elevadas temperaturas.

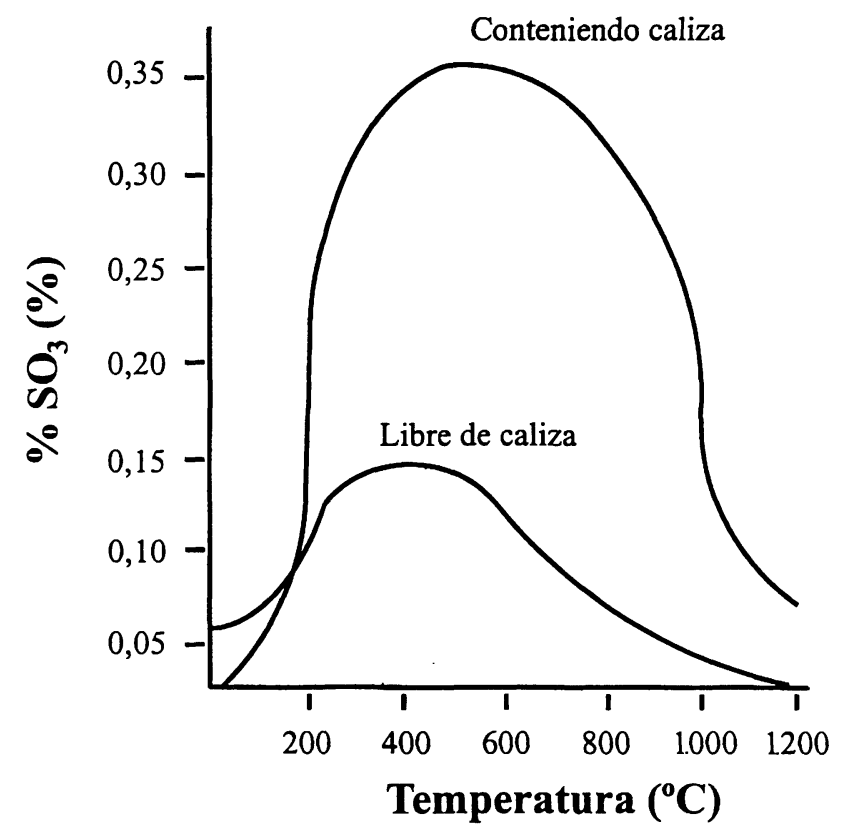

Figura 3.- Absorción de $\mathrm{SO}_{3}$ en el caso de una arcilla, con o sin contenido de cal (5).

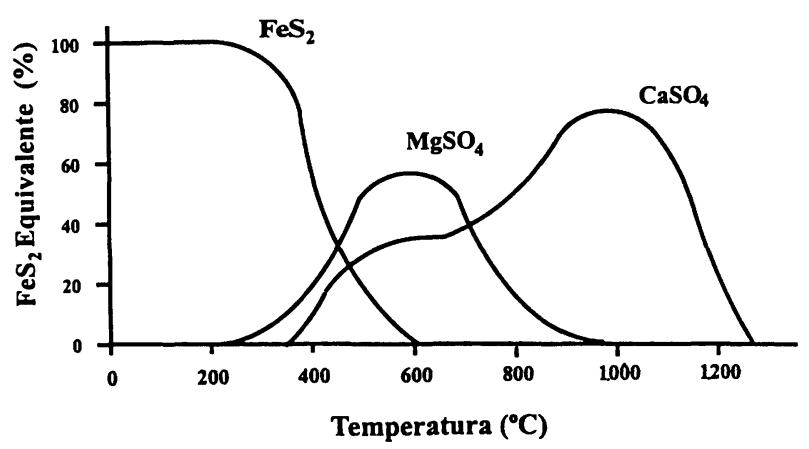

Figura 4.- Descomposición de la pirita y formación de sulfatos (según (5)).

\section{EJEMPLOS DE EFLORESCENCIAS MÁS FRECUENTES}

\subsection{DE SULFATO MAGNÉSICO $\left(\mathrm{SO}_{4} \mathrm{Mg}\right)$}

Es la eflorescencia más destructora, ya que se manifiestas como un desmoronamiento superficial que avanza progresivamente hacia el interior del ladrillo. Esta sal puede encontrarse como: 
- Solución en el agua de mar.

- $\mathrm{SO}_{4} \mathrm{Mg} \cdot \mathrm{H}_{2} \mathrm{O}$ monohidrato (kieserita o reichardita).

- $\mathrm{SO}_{4} \mathrm{Mg} \cdot 1 / 2 \mathrm{H}_{2} \mathrm{O}$ (sal de Epsom o de Sedlitz, epsomita).

- Sales dobles con $\mathrm{SO}_{4} \mathrm{Na}_{2}$, etc... (1).

Lo que más caracteriza al $\mathrm{SO}_{4} \mathrm{Mg}$ es su gran solubilidad en agua y su alta expansión al cristalizar. En los ladrillos cocidos puede haber $\mathrm{SO}_{4} \mathrm{Mg}$, que sufre el siguiente proceso al disolverse en agua y recristalizar.

$\mathrm{SO}_{4} \mathrm{Mg}+\mathrm{H}_{2} \mathrm{O}+$ cristalización $\longrightarrow \mathrm{SO}_{4} \mathrm{Mg} \cdot 7 \mathrm{H}_{2} \mathrm{O}$ $\left(P_{m}=120 ; \rho=2,7 \mathrm{~g} / \mathrm{cm}^{-3}\right) ; \quad\left(P_{m}=246 ; \rho=1,7 \mathrm{~g} / \mathrm{cm}^{-3}\right)$

\subsection{DE SULFATO DE SODIO $\left(\mathrm{SO}_{4} \mathrm{Na}_{2}\right)$}

Esta sal puede provocar fácilmente roturas (1), ya su eflorescencia ya que las presiones resultantes sobre la microestructura del ladrillo pueden llegar a alcanzar valores del orden de $25 \mathrm{~N} / \mathrm{mm}^{2}$ (5).

Esta eflorescencia puede formarse después de que el ladrillo haya sido puesto en obra. Así, la existencia de $\mathrm{CO}_{2}$ puede transformar el $\mathrm{SO}_{4} \mathrm{Ca}$ en $\mathrm{CO}_{3} \mathrm{Ca}$ a través de la reacción:

$\mathrm{SO}_{4} \mathrm{Ca}+\mathrm{MgO}+\mathrm{H}_{2} \mathrm{O}+\mathrm{CO}_{2} \rightarrow \mathrm{SO}_{4} \mathrm{Mg}+\mathrm{CO}_{3} \mathrm{Ca}+\mathrm{H}_{2} \mathrm{O}$

Este defecto, en obra, puede ser debido también a morteros ricos en $\mathrm{CO}_{3} \mathrm{Ca}$.

Esta sal puede producir también daños microestructurales a los ladrillos cuando cristaliza y recristaliza debido a fenómenos de expansión de volumen. En este caso se produce el decahidrato (5), dando lugar a presiones de cristalización del mismo orden que en el caso de la cristalización del sulfato magnésico hidratado.

$$
\mathrm{SO}_{4} \mathrm{Na}_{2}+\mathrm{H}_{2} \mathrm{O} \rightarrow \mathrm{SO}_{4} \mathrm{Na}_{2} \cdot 10 \mathrm{H}_{2} \mathrm{O}^{1}
$$

En este caso, la magnitud del daño depende de la localización del sulfato y es mayor si éste se sitúa en poros ocultos a la superficie del material.

\subsection{EFLORESCENCIAS DE VANADIO}

Aparecen en forma de manchas de color verde amarillento o amarillo de azufre y no se pueden eliminar por simple cepillado como las eflorescencias de sulfatos. Hay que

\footnotetext{
${ }^{1}$ No se han considerado a lo largo de esta revisión cuando las eflorescencias aparecen en el mortero o tienen su origen en el mismo. Se recuerda que esta revisión se refiere sólo a las eflorescencias directamente relacionadas con el material cerámico, sus materias primas o su proceso de fabricación.
}

tener en cuenta que no siempre que aparezcan manchas de colores pueden ser debidas a este tipo de eflorescencias. Cuando hay manchas coloreadas pueden existir también elementos tales como $\mathrm{Fe}, \mathrm{Mo}, \mathrm{Cu}, \mathrm{Cr}, \mathrm{Ni}$ y $\mathrm{Mn}$. Generalmente las eflorescencias de vanadio se deben a las materias primas o a los combustibles.

\section{DISTRIBUCIÓN DE LAS EFLORESCENCIAS EN OBRA}

Es frecuente observar que en obra la aparición de eflorescencias surge de forma irregular sobre las fábricas de los edificios. Este efecto se puede deber a agentes externos ajenos a los materiales, tales como:

* Ciclos térmicos anuales meteorológicos.

* Variaciones de la humedad relativa del aire.

* Variaciones de los vientos, etc...

Y también a la propia estructura del edificio, como:

* Salidas de tejados

* Calefacción del edificio

* Régimen de vientos en las calles

* Fugas de agua, etc...

Las variaciones locales en la intensidad de las eflorescencias pueden dar información sobre las causas que han motivado la extensión de este fenómeno. Pueden darse varios casos en obra en cuanto a la aparición de eflorescencias (5):

* Que afecten a toda la superficie de la cara vista de los ladrillos.

* Que afecten sólo a las juntas del mortero (o "llaga" de la fábrica de ladrillo).

* Que estén limitadas únicamente al borde de las juntas o de los ladrillos.

* Que tengan formas anulares o circulares en las caras de los ladrillos.

* Que aparezcan sólo en el centro de los ladrillos.

\section{CONCLUSIONES}

El fenómeno de aparición de eflorescencias en materiales de arcilla cocida es bien conocido desde hace tiempo y se ha visto, en la presente revisión, que hay datos abundantes en cuanto a tipos, métodos de estudio y corrección en el proceso. Sin embargo, sigue siendo un fenómeno al que se han hecho pocos estudios aplicando los más modernos métodos de análisis. El mayor problema es no sólo la complejidad del fenómeno, sino las múltiples e interferentes causas que hacen que se produzca esta patología en ladrillos. Recientemente, ha sido publicada por A. Verduch (6) una amplia monografia que 
recoge todo los conocimientos que se tienen hasta la fecha sobre este tema de gran interés no sólo para los fabricantes de materiales de construcción, sino también para los que aplican los ladrillos en obra.

\section{BIBLIOGRAFÍA}

(1) García-Verduch, A., Revista de Hyspalit (1966) 52-82.

(2) Jackson, F. G. , Amer.Cer.Soc.Bull. 4 (1925) 8, 376-401.
(3) AICE-ATC-IMPIVA, Tecnologia de la Fabricación de Pavimentos y Revestimientos, Castellón (1990).

(4) García-Verduch,A., I Congreso Iberoamericano de Cerámica, Vidrio y Refractarios, Ed. Soc.Esp.Ceram.Vidr., Madrid (1982) 577-584.

(5) Shmidt, CH.; Tile and Brick International, 9 (1993)4, 199-204.

(6) García-Verduch, A., Velos, Florescencias y Manchas en Obras de Ladrillo, Ed. Faenza Editrice Iberica, Castellón (1999).

\section{Publicación del Instituto Eduardo Torroja - CSIC}

\section{Número monográfico de INFORMES}

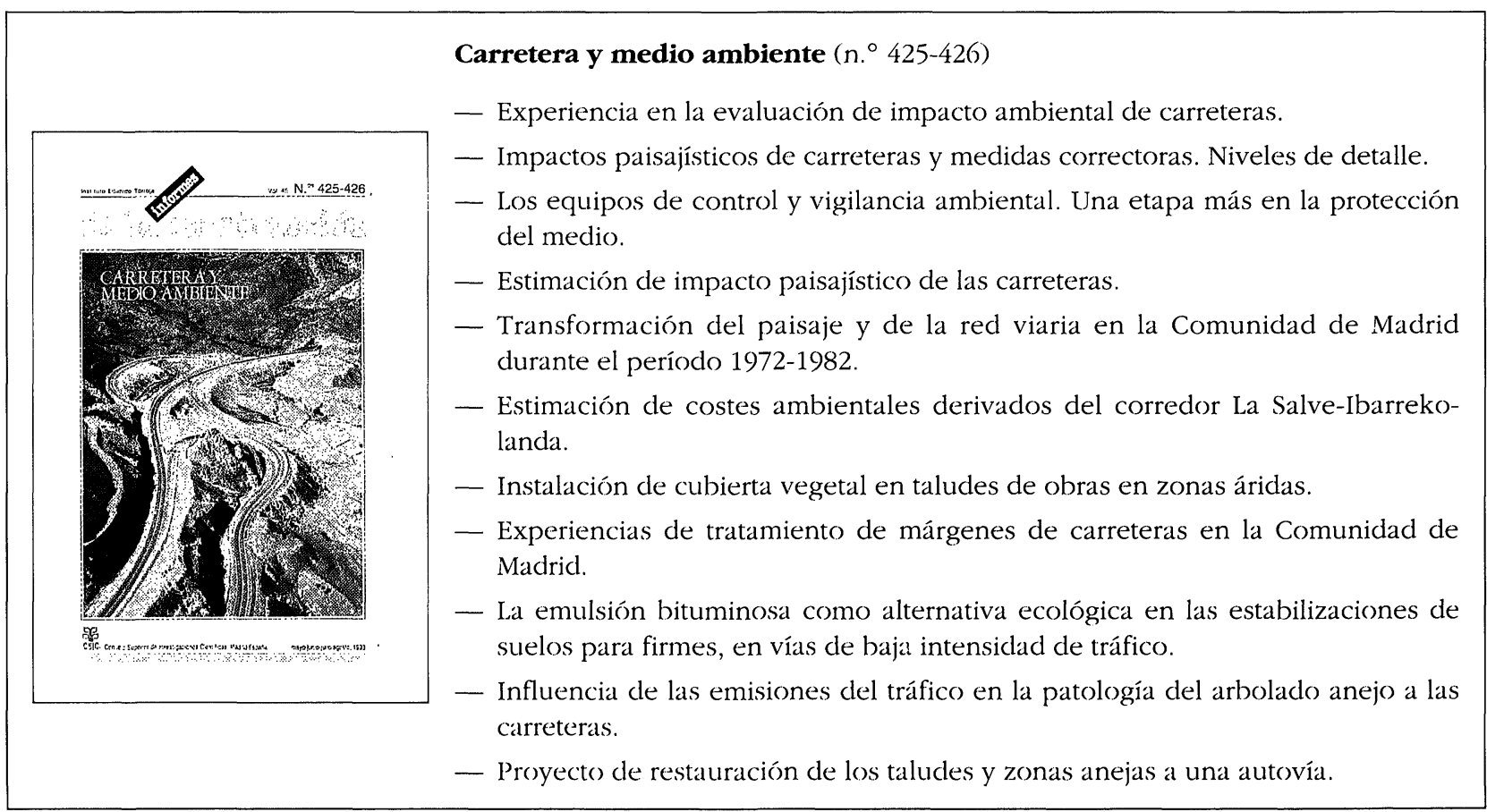

